\title{
Natural replacement of vertically inherited lux-rib genes of Photobacterium aquimaris by horizontally acquired homologues
}

\author{
Henryk Urbanczyk, ${ }^{1 *}$ Takashi Furukawa, ${ }^{1}$ \\ Yuki Yamamoto ${ }^{1,2}$ and Paul V. Dunlap ${ }^{3}$ \\ ${ }^{1}$ Interdisciplinary Research Organization, ${ }^{2}$ Faculty of \\ Medicine, University of Miyazaki, 5200 Kihara, Kiyotake, \\ Miyazaki 889-1692, Japan. \\ ${ }^{3}$ Department of Ecology and Evolutionary Biology, \\ University of Michigan, Ann Arbor, MI, USA.
}

\section{Summary}

We report here the first instance of a complete replacement of vertically inherited luminescence genes by horizontally acquired homologues. Different strains of Photobacterium aquimaris contain homologues of the lux-rib genes that have a different evolutionary history. Strain BS1 from the Black Sea contains a vertically inherited lux-rib operon, which presumably arose in the ancestor of this species, whereas the type strain NBRC $104633^{\top}$, from Sagami Bay, lacks the vertically inherited lux-rib operon and instead carries a complete and functional lux-rib operon acquired horizontally from a bacterium related to Photobacterium mandapamensis. The results indicate that the horizontal acquisition of the lux genes expanded the pan-genome of $P$. aquimaris, but it did not influence the phylogenetic divergence of this species.

\section{Introduction}

Bioluminescent bacteria are widespread in the marine environment and play important roles in marine ecosystems (Dunlap and Kita-Tsukamoto, 2006; Dunlap, 2009; Widder, 2010). Most marine luminous bacteria are members of Vibrionaceae and can be found in the genera Vibrio, Aliivibrio and Photobacterium (Baumann et al., 1984; Dunlap and Kita-Tsukamoto, 2006; Urbanczyk et al., 2007; 2011a; Ast et al., 2009; Dunlap, 2009). Bacterial luminescence is an activity coded for by the lux genes, which in Photobacterium are joined with the rib

Received 29 December, 2011; revised 7 March, 2012; accepted 25 April, 2012. *For correspondence. E-mail henryk@med.miyazakiu.ac.jp; Tel./Fax (+81) 985859764 .

(C) 2012 Society for Applied Microbiology and Blackwell Publishing Ltd genes to form a lux-rib operon (Lee et al., 1994; Lin et al., 2001; Ast et al., 2007; Urbanczyk et al., 2011a).

In addition to luminous members of Vibrionaceae, certain species of Enterobacteriaceae and Shewanellaceae are luminous (Jensen et al., 1980; Forst et al., 1997; Makemson et al., 1997; Dunlap and Kita-Tsukamoto, 2006; Waterfield et al., 2009). In most of these bacteria, the lux genes have been vertically inherited, whereas some species acquired these genes by horizontal transfer (Ast et al., 2007; Kasai et al., 2007; Urbanczyk et al., 2008; 2011a). The incidence of lux gene horizontal transfer appears to be rare, however, and in contrast to widely held views (e.g. Ochman et al., 2000; Gogarten et al., 2002) apparently has not led to speciation of the recipient strain based on current data (Urbanczyk et al., 2008).

The recent description of Photobacterium aquimaris, the described strains of which carry an apparently horizontally acquired luxA gene (Yoshizawa et al., 2009), provides a possible exception to the view that horizontal transfer of the lux genes does not contribute to divergence of the recipient. Analysis of $P$. aquimaris housekeeping genes revealed this species to be closely related to Photobacterium kishitanii and Photobacterium phosphoreum, whereas the IuxA gene apparently was acquired from a bacterium related to Photobacterium mandapamensis (Yoshizawa et al., 2009). In this study, we examined the evolutionary relationships of different strains of $P$. aquimaris to test the possibility that the phylogenetic divergence (i.e. divergence of lineages inferred from phylogenetic analysis of gene sequences) of this species was influenced by horizontal acquisition of the lux genes.

\section{Results and discussion}

Two strains of $P$. aquimaris, both thought to carry a horizontally acquired IuxA gene, have been described (Yoshizawa et al., 2009). To attempt to identify additional strains of this new species for analysis of the evolutionary origin of its luxA gene, we surveyed a wide diversity of luminous strains for new $P$. aquimaris isolates. Two luminous strains, BS1 and BS2, isolated from the Black Sea and provisionally identified as P. phosphoreum based on hybridization analysis (Wimpee et al., 1991), were 


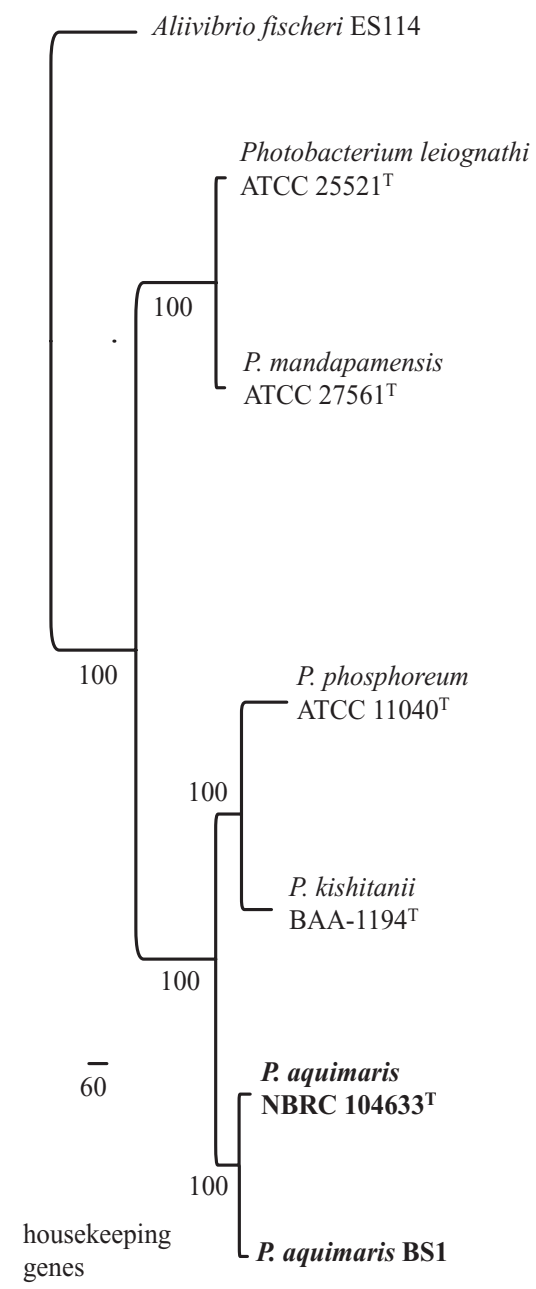

Aliivibrio fischeri ES114

Photobacterium leiognathi lelon.2.1 (lux-rib 2$)$

P. leiognathi lnuch.13.1 (lux-rib $\left.{ }_{2}\right) \mid 100$

P. mandapamensis ajapo.4.20 (lux-rib $\left.{ }_{2}\right)$

P. leiognathi ppana.3.2

P. leiognathi ppana.1.1

P. leiognathi lnuch.13.1 (lux-rib 1$)] 100$

P. leiognathi ATCC $25521^{\mathrm{T}}$

P. leiognathi ATCC 25587

P. leiognathi lelon.2.1 (lux-rib 1$)$

P. mandapamensis ajapo.2.19

P. mandapamensis ppana.3.7 100

P. mandapamensis gjord.1.5

P. mandapamensis sver. 1.2

P. mandapamensis ppana.3.10

P. mandapamensis sver.9.9

P. mandapamensis ATCC 33981 ,

P. mandapamensis ajapo.4.20 (lux-rib $\left.b_{1}\right)$

P. mandapamensis ajapo.4.5 100

P. mandapamensis seafl.1.1.

P. mandapamensis ajapo.5.1

P. mandapamensis PL-721

P. mandapamensis ATCC $27561^{\mathrm{T}}$

$P$. aquimaris NBRC $104633^{\mathrm{T}}$

P. phosphoreum ATCC $\left.11040^{\mathrm{T}}\right] 100$

$P$. phosphoreum $\mathrm{FS}-1$

P. phosphoreum AK4

P. phosphoreum AK8

$\overline{70}$

P. kishitanii ATCC BAA- $1194^{\mathrm{T}}$

P. kishitanii ckamo.1.1

P. kishitanii FS-8.1

P. aquimaris BS1 -100 lux genes

Fig. 1. Relationship between luminous Vibrionaceae based on housekeeping genes (left tree) and luxABFE genes sequences (right tree). Sequence data were analysed in PAUP* (Swofford, 2003) using the parsimony criterion. Jackknife support percentage values (after 1000 replicates) are shown at the nodes. The housekeeping genes were amplified as described in Ast and colleagues (2007), Urbanczyk and colleagues (2008) and Yoshizawa and colleagues (2009). Strains of $P$. aquimaris are shown in bold. For the housekeeping genes analysis, sequences of six genes, gyrB, pyrH, 16S, ftsZ, mreB and topA were concatenated and then aligned. The alignment had a total of 5362 characters (631 phylogenetically informative characters); the analysis resulted in a single most parsimonious tree. Analyses based on the individual genes were qualitatively similar to concatenations. Analyses of the concatenated housekeeping genes alignment were also carried out using neighbour-joining and maximum-likelihood algorithms, as done by Yoshizawa and colleagues (2009), and the results were congruent with the parsimony analysis (data not shown). For the luxABFE sequences analysis, only protein coding sequences were used. luxF, absent in Photobacterium leiognathi and Aliivibrio fischeri, was treated as missing data. The alignment had a total of 2495 characters (1106 phylogenetically informative characters), and the analysis resulted in two equally parsimonious trees. Photobacterium aquimaris strains are shown in bold. Roman numerals I and II refer to P. mandapamensis clades I and II respectively (Kaeding et al., 2007). Some jackknife support values were omitted for clarity. GenBank accession numbers for the sequences used in both analyses can be found in Table S1.

identified through analysis of housekeeping genes as likely members of $P$. aquimaris (data not shown), and one strain, BS1, was examined in greater detail here.

A multi-gene phylogenetic analysis based on housekeeping genes (16S rRNA gene, gyrB, ftsZ, mreB, pyrH and topA) revealed that $\mathrm{BS} 1$, like the type strain of $P$. aquimaris, NBRC $104633^{\top}$, is closely related to but distinct from P. phosphoreum and P. kishitanii (Fig. 1). However, analysis of the lux-rib operon genes (IuxABFE) of these bacteria revealed a different relationship (Fig. 1). Whereas the luxABFE genes of NBRC $104633^{\top}$ are phylogenetically affiliated with P. mandapamensis, as reported for luxA by Yoshizawa and colleagues (2009), the placement of the luXABFE genes of BS1 was concordant with the $P$. aquimaris housekeeping genes. We interpret this concordance as indicating that the lux-rib genes of BS1 were vertically inherited from the ancestor of $P$. aquimaris, which was luminous, whereas those of NBRC $104633^{\top}$ were horizontally acquired from a bacterium apparently closely related to $P$. mandapamensis.

To gain further insight into this issue, we cloned and sequenced the lux-rib operons of BS1 and NBRC $104633^{\top}$, 
P. aquimaris BS1

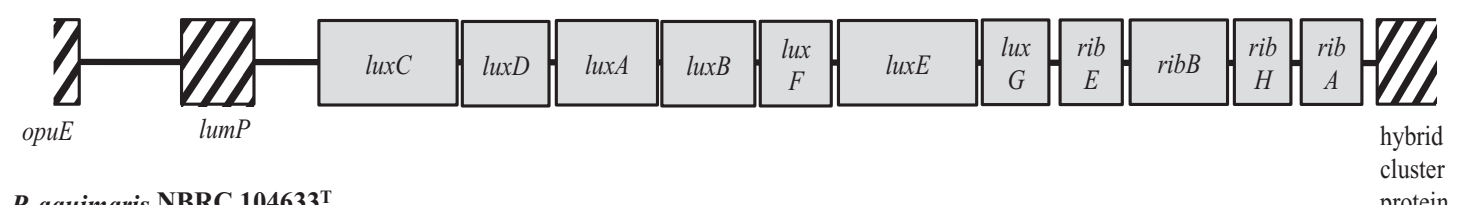

P. aquimaris NBRC $104633^{\mathrm{T}}$

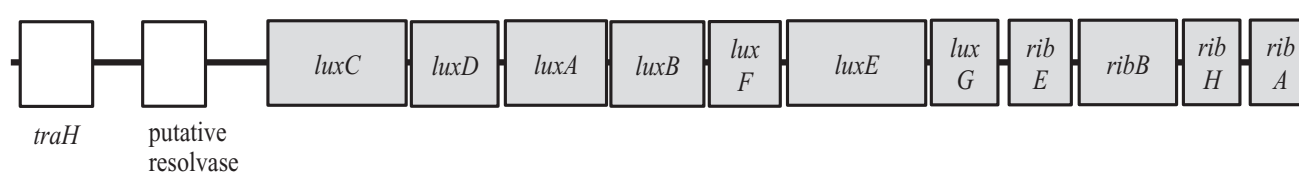

P. phosphoreum
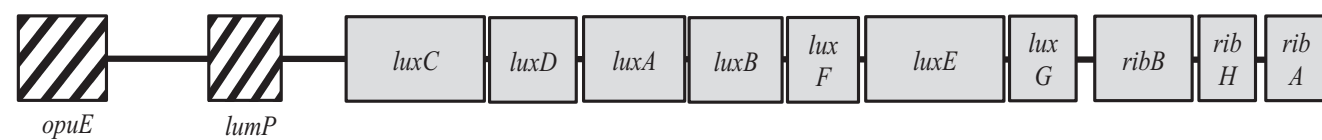

$1 \mathrm{~kb}$

Fig. 2. Gene organization of the lux-rib operons of $P$. aquimaris BS1 (upper panel), NBRC $104633^{\top}$ (middle panel) and P. phosphoreum (lower panel). The $P$. phosphoreum lux-rib operon gene organization was determined based on sequences of strains ATCC $11040^{\top}$ (GenBank accession number DQ988873) and NBRC 13896 (AB104437 and AB065117). Genes shaded grey are homologous, and hashed rectangles indicate sequences homologous between BS1 and P. phosphoreum. Cloning and sequencing of the genomic DNA followed the procedure described by Ast and colleagues (2007), except that genomic DNA cloned into pWEB-TNC cosmid (Epicentre) was sheared and the resulting fragments were religated into the pUC118-Hincll/BAP (Takara Biosciences) before sequencing.

including flanking DNA (Fig. 2). The genes upstream of the lux-rib operon of BS1 are homologous to a lumazine protein gene, lumP, and a proline transporter gene, opuE, which are also upstream of the luxC gene of $P$. phosphoreum (Kasai et al., 2007). Downstream of ribA in BS1 is a gene homologous to a hybrid cluster protein, which in P. phosphoreum is also located downstream of ribA (Kasai et al., 2007). This apparently conserved pattern of flanking genes suggests that the lux-rib operon is in the ancestral genomic position for these genes in the P. phosphoreum/ kishitaniilaquimaris clade. This conclusion was supported by phylogenetic analysis of complete lux-rib operons from representative luminous Photobacterium strains, including sequences of lux-rib operons of BS1 and NBRC $104633^{\top}$ (Fig. S1). In contrast, the genes upstream of the lux-rib operon of NBRC $10463^{\top}$ are a putative resolvase and a plasmid transport protein ( $t r a H$ ) (genes downstream of ribA were not recovered). The resolvase and $t r a H$ genes have no homology to sequences found in $P$. mandapamensis strain svers.1.1 genome (Urbanczyk et al., 2011b), or sequences flanking luminescence genes in other Vibrionaceae, and they have no role in light production. Instead, they apparently function in horizontal transfer of genes via superintegrons or plasmids (Hazen et al., 2010). The presence of these two genes upstream of luxC in NBRC $104633^{\top}$ is further support for horizontal acquisition of the lux-rib genes in NBRC $104633^{\top}$.

The results obtained here (Fig. 1) and previously (Yoshizawa et al., 2009) indicate that the source of the lux-rib genes of NBRC $104633^{\top}$ was likely to be a bacte- rium closely related to $P$. mandapamensis. Two phylogenetically distinct clades, I and II, however, comprise this species (Wada et al., 2006; Kaeding et al., 2007), and certain strains of this species acquired a second lux-rib operon, lux-rib $b_{2}$, by horizontal transfer (Ast et al., 2007; Urbanczyk et al., 2008). Therefore, the likely source of the NBRC $104633^{\top}$ lux-rib genes is not obvious. To attempt to identify which of these lineages might have been the source of these genes, we carried out a detailed sequence analysis of the luXABFE genes in luminous members of Vibrionaceae, including the horizontally transferred $l u x-r_{i} b_{2}$ operon of P. mandapamensis ajapo.4.20 (Fig. 1). The results confirm that the lux-rib operon of NBRC $104633^{\top}$ originated in bacterium closely related to extant members of $P$. mandapamensis, but the donor bacterium apparently belongs to neither clade I nor clade II. Furthermore, the analysis revealed that the horizontal transfer of lux-rib genes of NBRC $104633^{\top}$ was not a second example of the horizontal gene transfer (HGT) event that gave rise to the lux-rib merodiploidy of P. mandapamensis ajapo.4.20. These results indicate that the donor of the lux-rib operon of NBRC $104633^{\top}$ could belong to a previously unrecognized clade of $P$. mandapamensis that either has gone extinct or has not yet been sampled. Alternatively, sequence divergence observed in the analysis shown in Fig. 1 is a result of rapid lux-rib operon evolution in recipient $P$. aquimaris since the HGT event.

Only a single lux-rib operon was found in NBRC $104633^{\top}$ and BS1 in the amplification and sequencing work carried out here. Furthermore, attempts to PCR- 
amplify lux-rib sequences from NBRC $104633^{\top}$ genomic DNA with primers based on BS1 or from BS1 genomic DNA with primers based on NBRC $104633^{\top}$ were unsuccessful (data not shown). We also attempted to amplify lux-rib genes of NBRC $104633^{\top}$ and BS1 using specific primers designed for amplification of P. phosphoreum/ P. kishitanii or P. mandapamensis lux-rib operons (Ast et al., 2007), and all amplicons had the same sequence as the cloned lux-rib operons of each strain (data not shown). We also designed universal primers that can amplify luxA sequence in NBRC $104633^{\top}$, BS1, as well as in P. phosphoreum and P. mandapamensis. The product of PCR amplification with the universal primers always resulted in a single sequence of IUXA in all strains used. Cloning and analysis of seven random fragments of NBRC $104633^{\top}$ luxA amplified using universal primers also resulted in the same sequence (data not shown). These observations and the results presented above indicate that the horizontally acquired lux-rib genes completely replaced the vertically inherited luminescence genes in NBRC $104633^{\top}$. Whether this acquisition preceded, coincided with or followed loss of the vertically inherited genes is unknown.

In order to better understand the loss of the vertically inherited lux-rib operon from the NBRC $104633^{\top}$, we attempted to amplify sequences flanking the lux-rib operon of BS1 using the NBRC $104633^{\top}$ genomic DNA as a template. Only sequences located adjacent to ribA were amplified, but no sequences from the luxC side of the BS1 lux-rib operon were amplified (data not shown). Therefore, the mechanism under which the vertically inherited lux-rib operon of the NBRC $104633^{\top}$ was lost remains unknown. In this regard, however, gene replacement by homologues horizontally acquired from a distantly related species can reduce fitness of the recipient (Lind et al., 2010), so loss of the vertically inherited lux genes might have preceded their horizontal acquisition. Furthermore, there are several instances of strains lacking lux genes in otherwise luminous species of Vibrionaceae (Kaeding et al., 2007; O'Grady and Wimpee, 2008; Wollenberg et al., 2012). Therefore, it is possible that along with NBRC $104633^{\top}$, both non-luminous strains of $P$. aquimaris lacking the lux genes and luminous strains of this species that carry the vertically inherited lux genes exist. Regardless of those issues, we note that here as well as in previously described instances (Urbanczyk et al., 2008), the horizontal acquisition of the lux genes has expanded the pan-genome of $P$. aquimaris but has not led to phylogenetic divergence of this species.

\section{Conclusion}

The results presented here provide the first observation of a complete replacement of vertically inherited lumines- cence genes by horizontally acquired homologues. Different strains of $P$. aquimaris contain homologues of the lux-rib genes that have a different evolutionary history. Strain BS1 contains the vertically inherited lux-rib operon, which presumably arose in the ancestor of this species, whereas the type strain NBRC $104633^{\top}$ lacks the vertically inherited lux-rib operon and instead carries a complete and functional lux-rib operon acquired horizontally from a bacterium related to $P$. mandapamensis.

\section{Acknowledgements}

We thank C. Wimpee for the gift of strains BS1, BS2, AK4 and AK8. This work was financially supported by the Program to Disseminate Tenure Tracking System from the Japanese Ministry of Education, Culture, Sports, Science and Technology and by a grant for Scientific Research on Priority Areas from the University of Miyazaki.

\section{References}

Ast, J.C., Urbanczyk, H., and Dunlap, P.V. (2007) Natural merodiploidy of the lux-rib operon of Photobacterium leiognathi from coastal waters of Honshu, Japan. J Bacteriol 189: 6148-6158.

Ast, J.C., Urbanczyk, H., and Dunlap, P.V. (2009) Multi-gene analysis reveals previously unrecognized phylogenetic diversity in Aliivibrio. Syst Appl Microbiol 32: 379-386.

Baumann, P., Furniss, A.L., and Lee, J.V. (1984) Genus Vibrio Pacini 1854. In Bergey's Manual of Systematic Bacteriology. Kreig, N.R., and Holt, J.G. (eds). Baltimore, MD, USA: Williams and Wilkins, pp. 518-538.

Dunlap, P.V. (2009) Bioluminescence, microbial. In Encyclopedia of Microbiology. Schaechter, M. (ed.). Oxford, UK: Elsevier, pp. 45-61.

Dunlap, P.V., and Kita-Tsukamoto, K. (2006) Luminous bacteria. In The Prokaryotes: A Handbook on the Biology of Bacteria, Vol. 3. Dworkin, M., Falkow, S., Rosenberg, E., Schleifer, K.H., and Stackebrandt, E. (eds). New York, NY, USA: Springer, pp. 863-892.

Forst, S., Dowds, B., Boemare, N., and Stackebrandt, E. (1997) Xenorhabdus and Photorhabdus spp.: bugs that kill bugs. Ann Rev Microbiol 51: 47-72.

Gogarten, J.P., Doolittle, W.F., and Lawrence, J.G. (2002) Prokaryotic evolution in light of gene transfer. Mol Biol Evol 19: 2226-2238.

Hazen, T.H., Pan, L., Gu, J.D., and Sobecky, P.A. (2010) The contribution of mobile genetic elements to the evolution and ecology of Vibrios. FEMS Microbiol Ecol 74: 485499.

Jensen, M.J., Tebo, B.M., Baumann, P., Mandel, M., and Nealson, K.H. (1980) Characterization of Alteromonas hanedai (sp. nov.), a nonfermentative luminous species of marine origin. Curr Microbiol 3: 311-315.

Kaeding, A.J., Ast, J.C., Pearce, M.M., Urbanczyk, H., Kimura, S., Endo, H., et al. (2007) Phylogenetic diversity and cosymbiosis in the bioluminescent symbioses of 'Photobacterium mandapamensis'. Appl Environ Microbiol 73: 3173-3182. 
Kasai, S., Okada, K., Hoshino, A., lida, T., and Honda, T. (2007) Lateral transfer of the lux gene cluster. J Biochem 141: 231-237.

Lee, C.Y., O'Kane, D.J., and Meighen, E.A. (1994) Riboflavin synthesis genes are linked with the lux operon of Photobacterium phosphoreum. J Bacteriol 176: 2100-2104.

Lin, J.W., Chao, Y.F., and Weng, S.F. (2001) Riboflavin synthesis genes ribE, ribB, ribH, ribA reside in the lux operon of Photobacterium leiognathi. Biochem Biophys Res Commun 284: 587-595.

Lind, P.A., Tobin, C., Berg, O.G., Kurland, C.G., and Andersson, D.I. (2010) Compensatory gene amplification restores fitness after inter-species gene replacements. Mol Microbiol 75: 1061-1063.

Makemson, J.C., Fulayfil, N.R., Landry, W., Van Ert, L.M., Wimpee, C.F., Widder, E.A., and Case, J.F. (1997) Shewanella woodyi sp. nov., an exclusively respiratory luminous bacterium isolated from the Alboran Sea. Int $J$ Syst Bacteriol 47: 1034-1039.

Ochman, H., Lawrence, J.G., and Groisman, E. (2000) Lateral gene transfer and the nature of bacterial innovation. Nature 405: 299-304.

O'Grady, E.A., and Wimpee, C.F. (2008) Mutations in the lux operon of natural dark mutants in the genus Vibrio. Appl Environ Microbiol 74: 61-66.

Swofford, D.L. (2003) PAUP*: Phylogenetic Analysis Using Parsimony (*and Other Methods). Version 4. Sunderland, MA, USA: Sinauer Associates.

Urbanczyk, H., Ast, J.C., Higgins, M.J., Carson, J., and Dunlap, P.V. (2007) Reclassification of Vibrio fischeri, Vibrio logei, Vibrio salmonicida and Vibrio wodanis as Aliivibrio fischeri gen. nov., comb. nov., Aliivibrio logei comb. nov., Aliivibrio salmonicida comb. nov. and Aliivibrio wodanis comb. nov. Int J Syst Evol Microbiol 57: 28232829.

Urbanczyk, H., Ast, J.C., Kaeding, A.J., Oliver, J.D., and Dunlap, P.V. (2008) Phylogenetic analysis of the incidence of lux gene horizontal transfer in Vibrionaceae. J Bacteriol 190: 3494-3504.

Urbanczyk, H., Ast, J.C., and Dunlap, P.V. (2011a) Phylogeny, genomics, and symbiosis of Photobacterium. FEMS Microbiol Rev 35: 324-342.

Urbanczyk, H., Ogura, Y., Hendry, T.A., Gould, A.L., Kiwaki, N., Atkinson, J.T., et al. (2011b) Genome sequence of Photobacterium mandapamensis strain svers.1.1, the bioluminescent symbiont of the cardinal fish Siphamia versicolor. J Bacteriol 193: 3144-3145.

Wada, M., Kamiya, A., Uchiyama, N., Yoshizawa, S., KitaTsukamoto, K., Ikejima, K., et al. (2006) LuxA gene of light organ symbionts of the bioluminescent fish Acropoma japonicum (Acropomatidae) and Siphamia versicolor (Apogonidae) forms a lineage closely related to that of Photobacterium leiognathi ssp. mandapamensis. FEMS Microbiol Lett 260: 186-192.
Waterfield, N.R., Ciche, T., and Clarke, D. (2009) Photorhabdus and a host of hosts. Annu Rev Microbiol 63: 557574.

Widder, E.A. (2010) Bioluminescence in the ocean: origins of biological, chemical, and ecological diversity. Science 328: 704-708.

Wimpee, C.F., Nadeau, T.L., and Nealson, K.H. (1991) Development of species-specific hybridization probes for marine luminous bacteria by using in vitro DNA amplification. Appl Environ Microbiol 57: 1319-1324.

Wollenberg, M.S., Preheim, S.P., Polz, M.F., and Ruby, E.G. (2012) Polyphyly of non-bioluminescent Vibrio fischeri sharing a lux-locus deletion. Environ Microbiol 14: 655668.

Yoshizawa, S., Wada, M., Kita-Tsukamoto, K., Yokota, A., and Kogure, K. (2009) Photobacterium aquimaris sp. nov., a luminous marine bacterium isolated from seawater. Int $J$ Syst Evol Microbiol 59: 1438-1442.

\section{Supporting information}

Additional Supporting Information may be found in the online version of this article:

Fig. S1. Relationship between luminous Vibrionaceae based on housekeeping genes (left tree) and luxCDABFEGribEBHA genes sequences (right tree). The housekeeping tree was repeated from the Fig. 1 for reference. For the lux-rib sequences analysis, only protein coding sequences were used. Sequence data were analysed in PAUP* (Swofford, 2003) using the parsimony criterion. Treated as missing data were: luxF, absent in $P$. leiognathi and $A$. fischeri; ribE, absent in P. phosphoreum; and ribEBHA, absent in A. fischeri. The lux-rib sequence alignment had a total of 9453 characters, of which 2539 were phylogenetically informative. The analysis resulted in a single, most parsimonious tree, and analyses based on the individual genes gave the same phylogenetic placement. The trees were visualized using FigTree v. 1.3.1. Analyses of the housekeeping genes were also carried out using neighbour-joining and maximumlikelihood algorithms, as done by Yoshizawa and colleagues (2009), and the results were congruent with the parsimony analysis (data not shown). GenBank accession numbers for the sequences used in both analyses can be found in Table S1.

Table S1. GenBank accession numbers for sequences used in phylogenetic analyses. For A. fischeri ES114 gene locus tags from whole genome sequencing project (CP000020) were used.

Please note: Wiley-Blackwell are not responsible for the content or functionality of any supporting materials supplied by the authors. Any queries (other than missing material) should be directed to the corresponding author for the article. 